\title{
Influence of operational factors on the properties of working fluids of piston engines
}

\author{
Vladimir Zhukov ${ }^{1, *}$ and Olesya Melnik ${ }^{1}$ \\ ${ }^{1}$ Admiral Makarov State University of Maritime and Inland Shipping, St. Petersburg, Russia 198035
}

\begin{abstract}
The paper is devoted to the study of the regularities of changes in the quality of working fluids circulating through the systems of internal combustion piston engines. During the research, the parameters of fluids that characterize its operational properties, as well as external effects that can lead to changes in the properties of the fluid, were determined. It is shown that the deterioration of the quality of engine oil and coolant of piston engines during operation is caused by the destruction of additives that are part of the fluid and provide their required properties. A laboratory installation has been developed and created that allows simulating thermal and mechanical effects on working fluids similar to those acting during circulation through engine systems. The graphical dependencies obtained as a result of the experiment are presented. Mathematical processing of the experimental data allowed obtaining regression dependencies that correctly describe the processes of changes in time of the most important characteristics of working fluids under the action of cyclic thermal and mechanical effects. The described method can be used to study the influence of operational factors on the properties of various working fluids of internal combustion piston engines.
\end{abstract}

\section{Introduction}

Internal combustion piston engines (ICE) are the main element of power plants of road, rail and water transport, as well as stationary installations for various purposes $[1,2]$. The economic, resource and environmental performance of the internal combustion engine is influenced by both the operating conditions $[3,4]$ and the quality of operating materials, which include fuel, engine oil and coolant and the parameters of lubrication and cooling [5, 6]. The problems of improving the quality of operational materials due to the impact on their composition are solved in works $[7,8]$. The main method of improving the operational properties of working fluids is the introduction of additives into their composition - special substances in low concentrations.

Unlike fuel, the engine oils and coolants of the cooling system repeatedly circulate through the lubrication and cooling systems for a long time and should be considered as the working fluids of the engine. These fluids have a long service life, during which their composition changes, and their performance properties steadily deteriorate. Periodically, the engine oil and engine coolant fluid need to be replaced. The service life of the working

\footnotetext{
*Corresponding author: zhukovva@gumrf.ru
} 
fluids is determined by the engine manufacturers. During operation, the performance of the lubrication and cooling systems and the quality of working fluids are monitored, the methods and means of which are considered in works $[9,10]$. However, such control is not possible on all types of power plants, so it is of interest to predict the condition of working fluids, taking into account the impact of operational factors on them. The conducted research is devoted to this problem. Reasonable assignment of terms of replacement of operational materials ensures their rational use and reduces operating costs. To determine the permissible duration of use of engine oil and engine coolant fluid, it is necessary to investigate the influence of operational factors on their quality, characterized by a complex of physical and chemical properties.

\section{Methods and Materials}

External effects on the working fluids are caused by the processes that accompany their circulation through the internal combustion engine systems. The main effects are associated with changes in temperature and pressure in the systems and can be considered as thermal and mechanical factors that affect, first of all, the additives contained in the working fluids.

To simulate the thermal effect, it is necessary to carry out cyclic heating of the fluid in the temperature range from 20 to $100^{\circ} \mathrm{C}$, typical for lubrication and cooling systems, followed by cooling, which reproduces the engine start, warm - up, subsequent stop and cooling. The heating intensity must be such that the temperature rise time corresponds to the engine warm-up time under operating conditions.

The nature of the thermal effect on the working fluid is shown in figure 1.

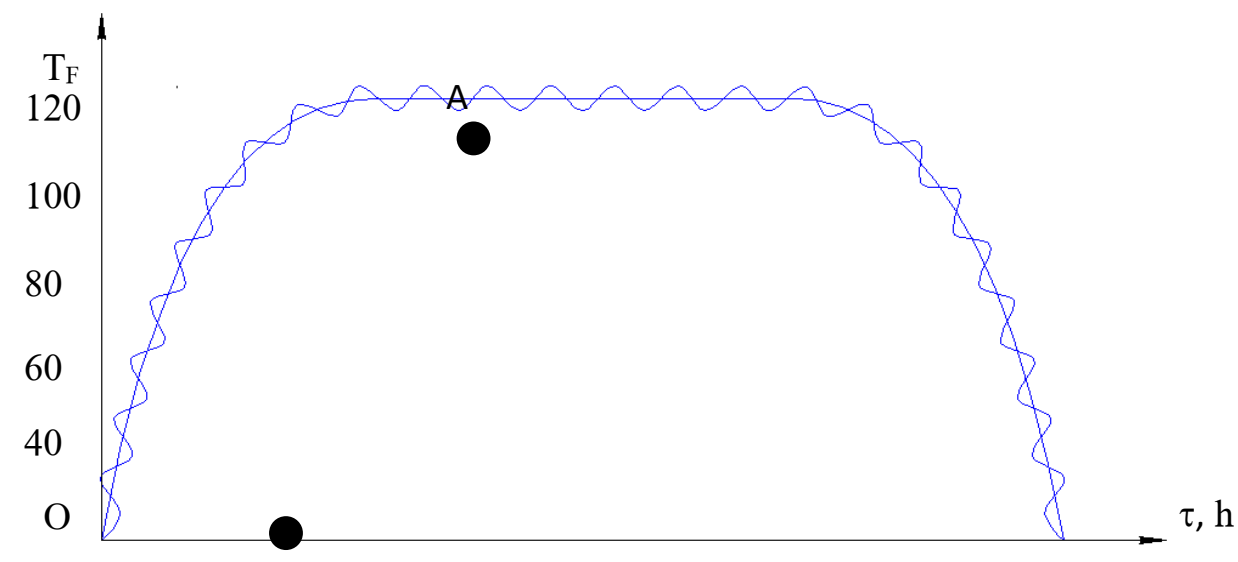

Fig. 1. Change in the temperature of the working fluid during the operation of the engine.

Macro-temperature changes simulate the process of warming up the engine after starting and cooling after stopping, micro-changes correspond to temperature fluctuations in the process of circulation through the cooling system. The speed of reaching the steady-state thermal regime (OA section) characterizes the intensity of the thermal effect on the fluid. The unit of measurement of the intensity of thermal effect can be the value of ${ }^{\circ} \mathrm{C} / \mathrm{min}$. The duration of effect can be measured in engine-hours or the number of macro-heating cycles.

The mechanical effect on the working fluids occurs during their pumping through the lubrication and cooling systems. The circulation speed is in the range from 1 to $5 \mathrm{~m} / \mathrm{s}$ in the systems of existing engines. At the same time, there are stagnant zones in which the fluid velocity is only $0.1 \ldots 0.3 \mathrm{~m} / \mathrm{s}$. These velocities correspond to the turbulent and laminar flow modes. Thus, when conducting research, it is necessary to reproduce both characteristic 
flow modes with Reynolds numbers less than 1000 and more than 10000, while maintaining the speed in the real range.

The mechanical effects on the fluid are illustrated in figure 2.

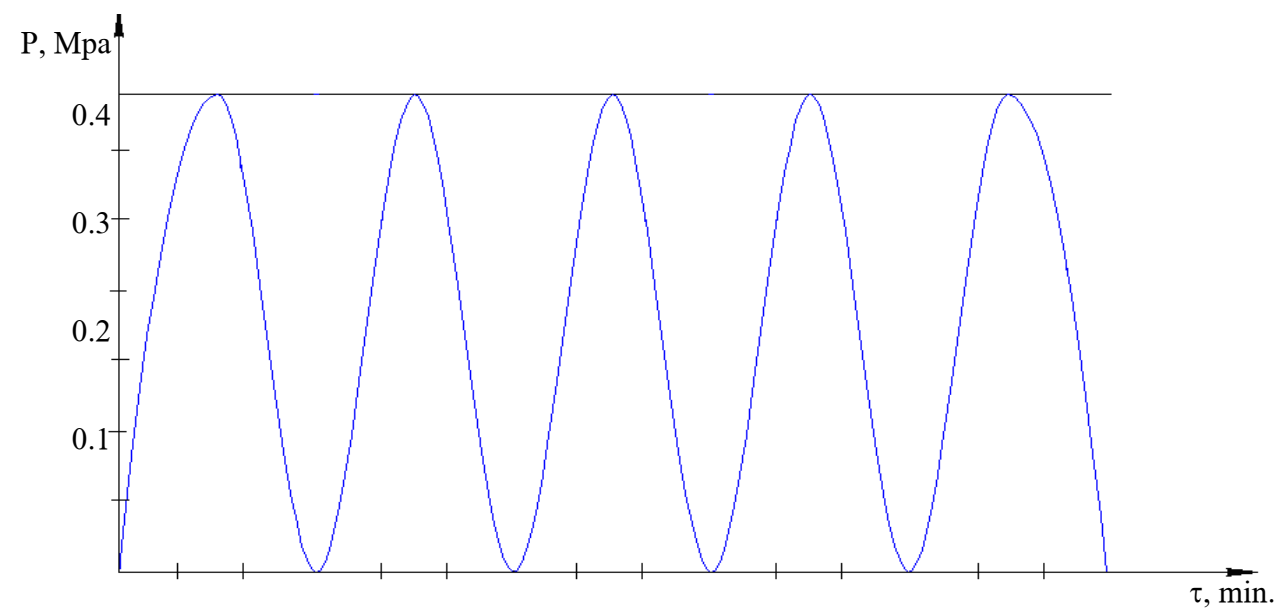

Fig. 2. Mechanical effects on the fluid during circulation.

The amplitude of the oscillations (figure 2) corresponds to the maximum hydraulic resistance of the circulation loop, and their period is determined by the speed of fluid circulation.

By changing these parameters in the ranges characteristic of real cooling systems, it is possible to determine their influence on the aging processes of coolant fluids containing additives and to predict the timing of reaching the limit values of operational indicators and develop recommendations for their maintenance within the established limits.

To simulate thermomechanical effects on the fluid, a laboratory installation was created, the scheme of which is shown in figure 3.

The object of research was fluids containing substances that are part of multifunctional additives to coolant fluid of the internal combustion engine, which effectively inhibit cavitation and corrosion damage characteristic of elements of piston internal combustion engine systems [11-14], and prevent scale formation on the cooled surfaces and the additives themselves in working concentrations. Untreated distilled water was used as the base fluid for the tests. The composition of the additive includes substances that can change the structure of the wall layer of the fluid and significantly affect the intensity of heat exchange. Such substances include, first of all, the water-soluble polymer polyacrylamide (PAA), the surfactant Syntanol DS-10 (surfactant), sodium silicate, which is used as the main corrosion inhibitor.

The purpose of the research was to determine the dynamics of changes in the properties of fluids with additives that affect both the processes of cavitation-corrosion destruction and the processes of heat exchange. When developing the research methodology, it was taken into account that the most characteristic for modern forced engines are the processes of heat exchange with bubble boiling on the most heated surfaces of parts. 


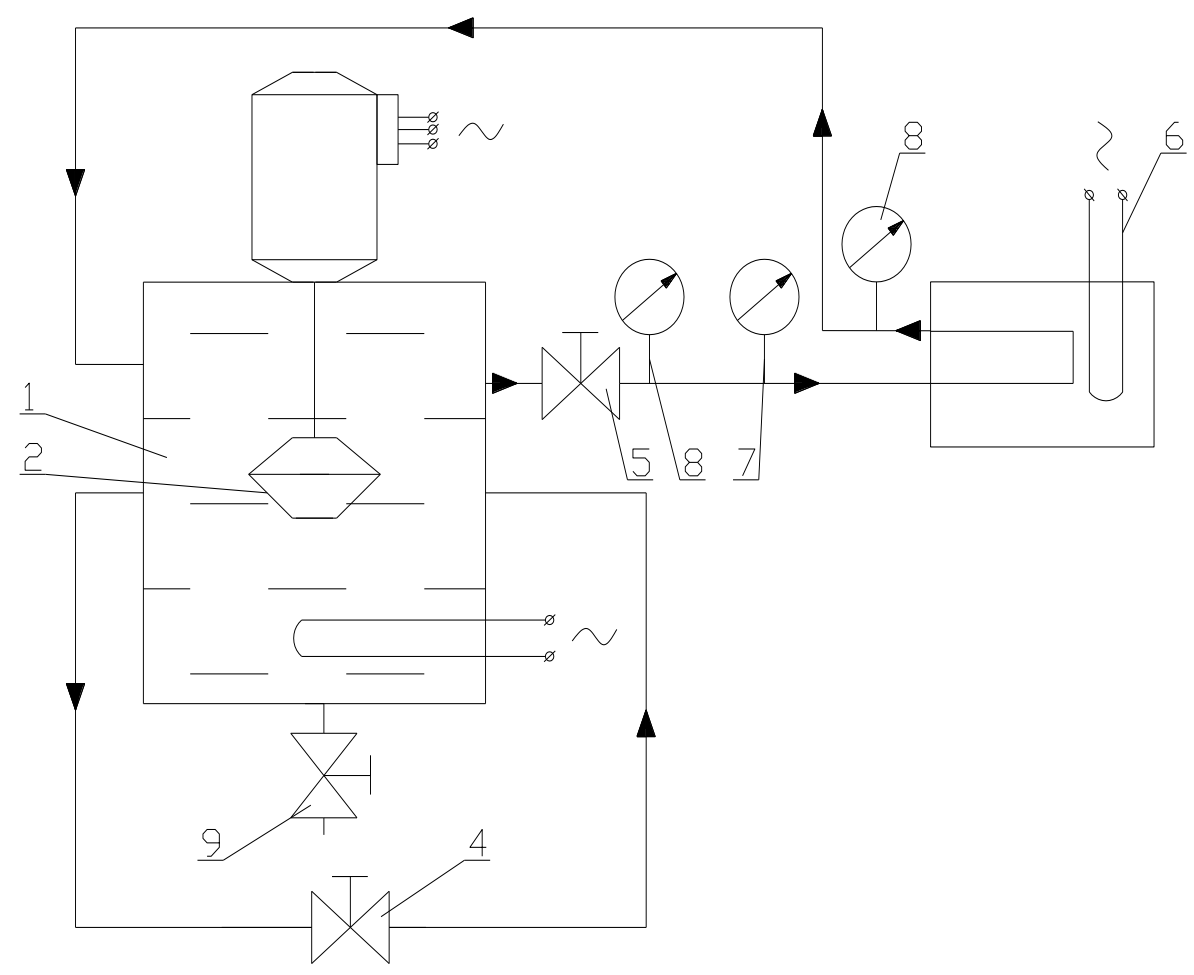

Fig. 3. Diagram of the laboratory installation. Where: 1 - fluid reservoir, equipped with a cooler, $2-$ circulation pump, 4 - bypass valve serving to regulate the flow rate and speed of fluid circulation, 5 pressure reducing valve designed to change the hydraulic resistance of the circuit, 6 - heater to ensure a given level of temperature of the fluid, 7 - thermometer, 8 - differential pressure gauge, 9 - drain valve used for sampling fluid.

Introduction to water of additives and components included in them in concentrations of not more than $0.5 \%$ by weight, is not able to change such properties of the fluid as density, heat capacity or thermal conductivity, which is why the surface tension, which is one of the most important factors determining the processes of bubble boiling, was chosen as a parameter characterizing the thermos-physical properties of the fluid.

It was decided to use the hydrogen index $(\mathrm{pH})$ and the specific electrical conductivity (EC) of the fluids as the parameters characterizing the corrosion aggressiveness of the coolant fluids.

To determine the hydrogen index, the MULTITEST IPL-513 fluid analyzer was used.

The specific electrical conductivity was determined using the conductometric method by conductometer MULTITEST KSL-101.

The viscosity of the fluid characterizes the power required to pump it through the circulation loop and can be used to assess the degree of degradation of additives.

The viscosities of the studied fluids were measured at test temperatures with a capillary viscometer.

\section{Results}

Samples of fluids taken after certain periods of time were analyzed using these devices in order to determine changes in the performance characteristics of fluids. 
The results of processing experimental data on the determination of the hydrogen index $(\mathrm{pH})$, surface tension $(\sigma)$, and viscosity $(v)$ of fluids with various additives are presented in the form of graphical dependencies in figure 4.

The dotted line on the graphs corresponds to the indicators of water without additives, which was used as a reference.

The analysis of the graphs shows that the introduction of additives provides an increase in the hydrogen index, the largest increase in the hydrogen index is 11 . Thermal and thermomechanical effects on fluids in all the experiments conducted lead to a decrease in the hydrogen index. At the same time, it should be noted:

- The hydrogen index of the sodium silicate solution remains the most stable under various effects;

- The least stable is the hydrogen index of the surfactant solution, which indicates the intensive destruction of micellar formations of surfactants under the influence of temperature and mechanical effects;

- Decrease in the hydraulic resistance of the circulation loop and the inevitable increase in the flow speed lead to a more intense decrease in the hydrogen index;

- Increasing the concentration of the additive increases the initial value of the hydrogen index and slows its fall.

Characteristic changes in the hydrogen index indicate a deterioration in the protective properties of additives. The values of the hydrogen index can be used to monitor the condition of the coolant fluid.

The nature of the change in the surface tension coefficient of the studied fluids allows drawing the following conclusions:

- the introduction of $0.1 \%$ by weight of sodium silicate reduces the surface tension compared to distilled water by $20 \%$, but after three hours of bubble boiling, surface tension increases to values typical for water and then remains stable;

- the introduction of $0.1 \%$ by weight of PAA leads to a decrease in the surface tension coefficient by about $15 \%$, during five hours of thermal effect, the surface tension increases and stabilizes at a value slightly higher than the indicator for water;

- the introduction of $0.1 \%$ surfactant reduces the surface tension of the initial solution by $25 \%$ compared to water. The effect by bubble boiling leads to the fact that the surface tension coefficient decreases and after 10 hours it stabilizes at a value equal to 0.5 of the value for distilled water. This indirectly confirms the numerous available experimental data on the ability of surfactants to significantly intensify heat exchange in the presence of phase transitions and allows making an assumption about the long-term effect of surfactants on heat exchange processes. 

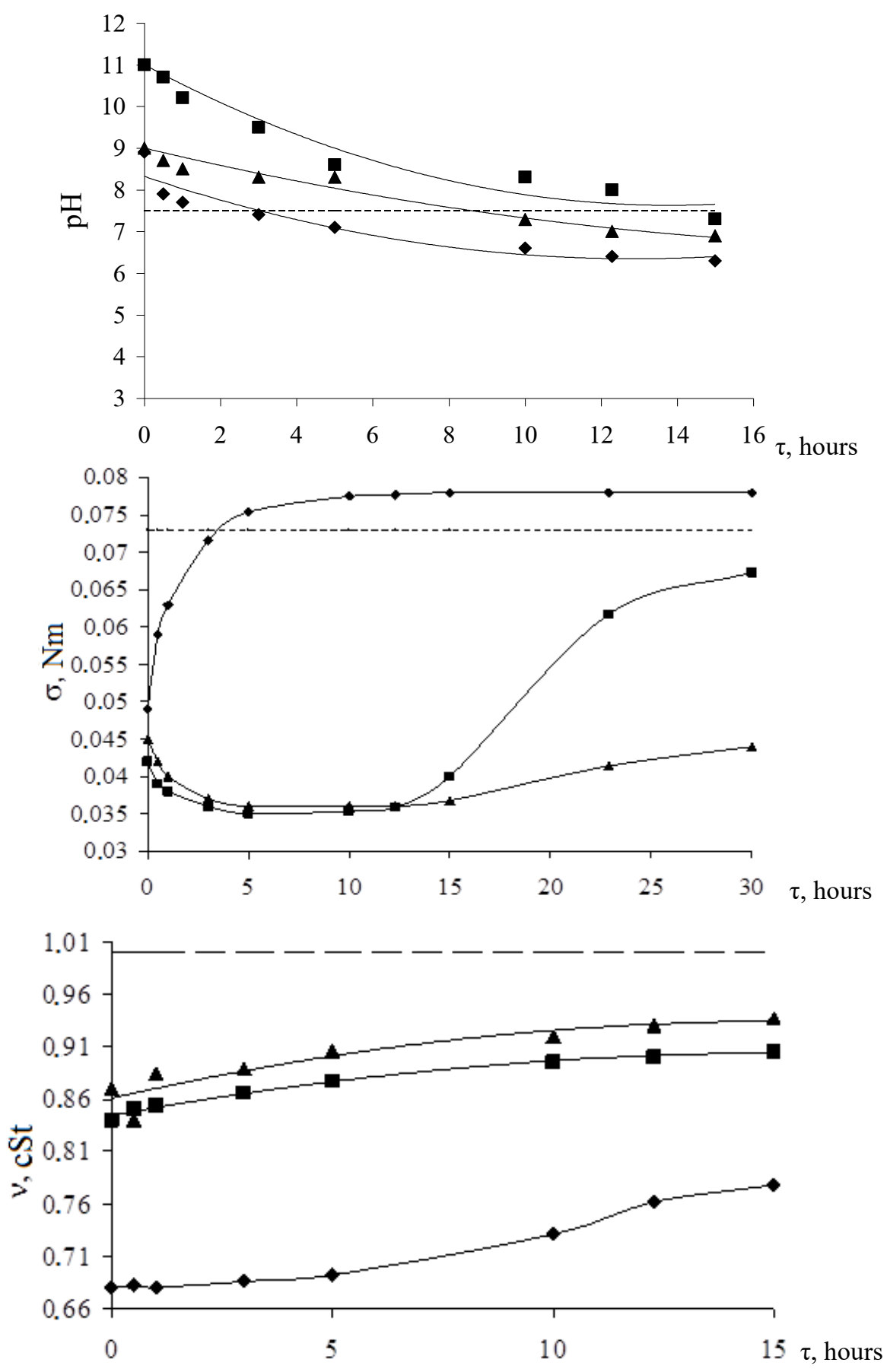

Fig. 4. Changes in the physical and chemical characteristics of fluids under the action of thermal and mechanical effects: $\downarrow$ - sodium silicate, $\boldsymbol{-}$ - surfactant, $\boldsymbol{\Lambda}$ - PAA.

Laboratory studies have confirmed the ability of additives to change the surface tension of the fluid and the gradual reduction of this change under the action of thermal and thermomechanical effects. These facts allow using the surface tension coefficient as a 
parameter for diagnosing the condition of the coolant of the cooling system during operation.

Measurements of the viscosities of the initial fluids showed that the most noticeable effect on the viscosity is the introduction of surfactants and PAA, which reduce the viscosity of distilled water by $15-20 \%$ (for experimental concentrations).

The results obtained allow drawing the following conclusions:

- the introduction of individual components of the additive and their composition provides a decrease in viscosity. The composition of the additive, which contains all the components, allows getting the greatest reduction in viscosity - up to 0.81 . Thermal and thermomechanical effects on fluids in all the experiments conducted lead to an increase in the viscosity value. However, it should be noted:

- the viscosity of the polyacrylamide solution remains the most stable under various effects;

- changing the hydraulic resistance of the circulation loop leads to a change in the speed of destruction of the additive;

- $\quad$ increasing the additive concentration lowers the initial viscosity value.

The viscosity values can be used to develop recommendations for the use of additives and to maintain the established properties of coolants in operation.

Studies of the specific electrical conductivity of fluids containing additives in various concentrations (figure 5) showed that the introduction of the additive provides a significant increase in this indicator, and, with an increase in the concentration of the additive from 0.3 to $0.6 \%$, the initial specific electrical conductivity of the fluid increases from 1.7 to $2.1 \mathrm{mS}$. Thermal and thermomechanical effects on the fluid led to almost the same results: the specific electrical conductivity increases intensively in the initial period of time, then the growth gradually slows down and asymptotically tends to a certain constant value. These changes can be explained as follows. A sharp increase in specific electrical conductivity with the introduction of additives (almost $10^{3}$ compared to distilled water) is caused by the presence in the solution of alkaline bases and ions formed during the dissolution of sodium silicate, PAA and surfactants. The subsequent increase in specific electrical conductivity is associated with an increase in the concentration of particles that provide electrical conductivity as a result of the destruction of polymer macromolecules and micellar formations of surfactants.

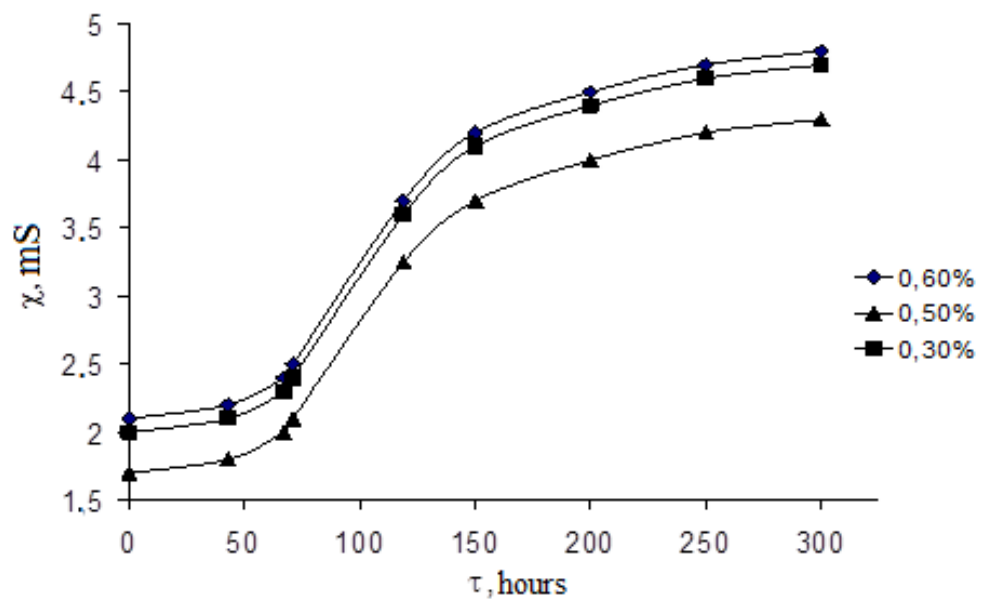

Fig. 5. Dependence of the specific electrical conductivity $\chi$ of additive solutions of different concentrations on the time of thermomechanical effect $\tau$. 
During the tests, it was not possible to establish the limit value of the specific electrical conductivity, and the correlation of this indicator with the operational properties of coolants, so the use of specific electrical conductivity as a rejection indicator that characterizes the chemical aggressiveness of the coolant fluid does not seem appropriate.

\section{Discussion}

The results of the experiments were used to construct regression dependences of the hydrogen index, surface tension, and kinematic viscosity of the coolant fluid on operational factors. Each of the samples was subjected to three parallel tests, according to which the average values of the measurements were calculated $\overline{p H}, \bar{\sigma}, \bar{v}$.

The free term of the regression dependence was calculated by the equation:

$$
b_{0}=\frac{1}{\mathrm{~N}} \sum_{j=1}^{\mathrm{N}} \bar{y}_{j}
$$

where $\overline{y_{j}}-$ the arithmetic mean for all experiments at the main level; $\mathrm{N}-$ the total number of experiments in the planning matrix.

The coefficient before the $i$-th factor was calculated by the equation:

$$
b_{i}=\frac{1}{\mathrm{~N}} \sum_{j=1}^{\mathrm{N}} x_{i j} \bar{y}_{j} \text {. }
$$

The value of the coefficient indicates the strength of the impact factor: the larger the coefficient, the greater the effect on the response function has a corresponding factor. If the coefficient is positive -then with an increase of a factor increasing the amount of the response function, if the coefficient is negative, then the magnitude of the response function is reduced. The arguments of the regression dependences are operational factors: pressure in the system $\Delta \mathrm{p}$, temperature $\mathrm{T}$, and time $\tau$.

The regression equation for the hydrogen index has the form:

$$
p H=6.425+5.17 \cdot 10-3 \Delta \mathrm{P}+1.5 \cdot 10-3 \tau+33.0 \cdot 10-3 T .
$$

The regression equations for surface tension has the form:

$$
\sigma=1.678-3.41 \cdot 10-4 \Delta \mathrm{P}-2.36 \cdot 10-4 \tau-0.0175 \mathrm{~T} \text {. }
$$

Regression equation of kinematic viscosity has the form:

$$
v=1.975-10.5 \cdot 10-2 \Delta \mathrm{P}+6.5 \cdot 10-3 \tau+3.5 \cdot 10-3 \mathrm{~T} \text {. }
$$

Verification of the adequacy of the obtained equations by the Fisher criterion confirmed their adequacy.

\section{Conclusion}

As a result of the conducted research, the analysis of the main operational indicators of working fluids of piston engines and the factors that cause changes in these indicators during operation is carried out.

A laboratory installation has been created that allows simulating thermos-physical effects on working fluids similar to operational ones. Based on the results of the 
experiments, regression dependences are obtained that describe the change in the hydrogen index $p H$, surface tension $\sigma$, and viscosity $v$ of the fluid depending on the pressure drop in the system $\Delta p$, temperature $\mathrm{T}$, and time $\tau$.

Based on the results of checking the equations using the Fisher criterion, it can be concluded that the regression equations are adequate and can be used to assess the influence of the factors $\Delta p, \tau, T$ on the main characteristics of the fluid that determine its operational properties.

The values of the coefficients in the obtained regression equations allow drawing conclusions about the nature of the influence of the factors under consideration on the operational properties of the fluid.

For fluid viscosity, the most significant factor is the hydraulic resistance of the system. This is due to the fact that with a decrease in resistance, the speed of fluid circulation increases, the turbulence of the flow increases, and the orientation of polymer macromolecules and micellar formations of surfactants in the wall areas occurs, as a result of which the effect of additives on reducing the viscosity becomes more noticeable.

The influence of the fluid temperature is several times stronger than the influence of the hydraulic resistance of the system and the duration of operation on the change in the hydrogen index and orders of magnitude stronger than their influence on the change in the surface tension. The significant influence of temperature can be explained on the basis of the thermal fluctuation nature of the destruction.

The laboratory installation and the experimental technique used to study the patterns of changes in the properties of the coolant of the internal combustion engine cooling system can also be used to study the processes of changing the quality of the engine oil of the internal combustion engine during operation.

\section{References}

1. Internal Combustion Engines: Performance, Fuel Economy and Emissions (IMechE, Woodhead Publishing, 2013)

2. Doug Woodyard, Pounder's Marine Diesel Engines And Gas Turbines (ButterworthHeinemann, 2014)

3. S. Gaidar, M. Karelina, A. Laguzin, Hoang Duc Quang, Transportation Research Procedia 50, 136-144 (2020) doi: 10.1016/j.trpro.2020.10.017

4. V. Baskov, A. Ignatov, V. Polotnyanschikov, Transportation Research Procedia 50, $37-$ 43 (2020) Doi: 10.1016/j.trpro.2020.10.005

5. V.A. Zhukov, O.V. Melnik, L.V. Tuzov, J. GUMRF named Admiral S. O. Makarov St. Petersburg 5(51), 1040-1051 (2018)

6. H. Moussa Nahim, R. Younes, H. Shraim, M. Ouladsine, IFAC-PapersOnLine 49(11), 570-575 (2016) Doi:10.1016/j.ifacol.2016.08.083.

7. Mohammad Hemmat Esfe, Ali Akbar Abbasian Arani, Saeed Esfandeh, Masoud Afrand, Energy 170, 228-23 (2019) Doi: 10.1016/j.energy.2018.12.127.

8. Mohammad Hatami, Maryam Hasanpour, Dengwei Jing, Journal of Molecular Liquids 318, 114250 (2020)

9. J.M. Wakiru et al., Mechanical Systems and Signal Processing 118, 108-132 (2019) doi: 10.1016/j.ymssp.2018.08.039

10. X. Zhu, C. Zhong, J. Zhe, Tribology International 109, 473-484 (2017) doi: 10.1016/j.triboint.2017.01.015

11. Feng Cheng, Weixi Ji, Chenhao Qian, Xu Jie, Results Phys 9, 1585-1593 (2018) 
12. M. Brunhart, C. Soteriou, C. Daveau, M. Gavaises, M. Winterbourn, Wear 442-443, 203024 (2020) doi: 10.1016/j.wear.2019.203024

13. M. Cristofaro, W. Edelbauer, P. Koukouvinis, M. Gavaises, Applied Mathematical Modelling 78, 200-216 (2020) doi: 10.1016/j.apm.2019.09.002

14. Tengfei Cai, Yan Pan, Fei Ma, Experimental Thermal and Fluid Science 1171, 110137 (2020) doi: 10.1016/j.expthermflusci.2020.110137 